\title{
Sound propagation in saturated gas-vapor-droplet suspensions considering the effect of transpiration on droplet evaporation
}

\author{
Max Kandula \\ ESC - Team QNA, NASA Kennedy Space Center, FL 32899, USA \\ max.kandula-1@nasa.gov
}

\begin{abstract}
The Sound attenuation and dispersion in saturated gas-vapor-droplet mixtures with evaporation has been investigated theoretically. The theory is based on an extension of the work of Davidson (1975) to accommodate the effects of transpiration on the linear particle relaxation processes of mass, momentum and energy transfer. It is shown that the inclusion of transpiration in the presence of mass transfer improves the agreement between the theory and the experimental data of Cole and Dobbins (1971) for sound attenuation in air-water fogs at low droplet mass concentrations. The results suggest that transpiration has an appreciable effect on both sound absorption and dispersion for both low and high droplet mass concentrations.
\end{abstract}

\section{Nomenclature}

$c_{0} \quad=$ sound speed in undisturbed (particle-free) gaseous mixture (air and water vapor), $\sqrt{\gamma R_{0} T_{0}}$

$c=$ sound velocity in mixture

$c_{p} \quad=$ mixture specific heat at constant pressure

$c_{p} \quad=$ mixture specific heat at constant volume

$c_{p p} \quad=$ specific heat of droplet at constant pressure

$C_{D} \quad=$ droplet drag coefficient, $F_{p} /\left[\frac{1}{2} \rho \frac{\pi}{4} d_{p}^{2}\left(u_{p}-u\right)^{2}\right]$

$C_{m} \quad=$ droplet mass loading (ratio of mass of droplets to mass of gaseous mixture per unit volume of undisturbed mixture-droplet system, $\rho_{m 0} / \rho_{0}=n_{0} m_{p} / \rho_{0}$ 
$C_{v} \quad=$ vapor mass loading (ratio of mass of vapor to mass of gaseous mixture per unit volume of undisturbed mixture-droplet system, $\rho_{v 0} / \rho_{0}$

$d_{p} \quad=$ droplet diameter

$D \quad=$.mass diffusivity of the gaseous mixture

$f \quad=$ frequency of sound

$F_{p} \quad=$ drag force between the mixture and a single droplet, $\frac{\pi}{8} C_{D} \rho d_{p}^{2}\left(u_{p}-u\right)^{2}$

$h \quad=$ convective heat transfer coefficient between the mixture and a single droplet

$h_{l}=$ latent heat of vaporization, $h_{L 0}+\left(c_{p v}-c_{p p}\right)\left(T_{p}-T_{0}\right)$

$h_{m} \quad=$ convective mass transfer coefficient between the mixture and a single droplet

$H_{g} \quad=$ specific heat ratio of gas, $c_{p g} / c_{p 0}$

$H_{p} \quad=$ specific heat ratio of particles, $c_{p p} / c_{p 0}$

$H_{v} \quad=$ specific heat ratio of particles, $c_{p v} / c_{p 0}$

$k=$ mixture thermal conductivity

$K=$ complex wave number, $k_{1}+i k_{2}$

$L \quad=$ latent heat parameter (dimensionless), $h_{L 0} / R_{\nu} T_{0}$

Le $\quad=$ Lewis number, $k / \rho c_{p} D$

$m_{p} \quad=$ mass of one droplet

$M_{p} \quad=$ diffusional mass transfer between the mixture and a single droplet, $\pi d_{p} D S h\left(\rho_{s}-\rho_{v}\right)$

$n_{0} \quad=$ mean number of droplets per unit volume of mixture

$\mathrm{Nu}=$ droplet Nusselt number, $h d_{p} / k_{0}$

$p \quad=$ mixture pressure

$\operatorname{Pr} \quad=$ Prandtl number, $c_{p 0} \mu_{0} / k_{0}$

$Q_{p} \quad=$ heat transfer between the mixture and a single drop, $\pi d_{p} k N u\left(T_{p}-T\right)$

$R \quad=$ ideal gas constant of mixture (instantaneous), $R_{0}\left(1+R^{\prime}\right)$

$\operatorname{Re}_{p} \quad=$ droplet Reynolds number, $\rho_{0}\left|u_{p}-u\right| d_{p} / \mu_{0}$

Sh $=$ droplet Sherwood number, $h_{m} d_{p} / D_{0}$

Sc $=$ Schmidt number, $v_{0} / D_{0}=L e \operatorname{Pr}$

$S=$ Spalding transport parameter

$T=$ mixture temperature

$u=$ mixture velocity in the $x$-direction

$x=$ coordinate distance 


\section{Greek Symbols}

$\alpha \quad=$ sound (energy) absorption coefficient (dimensionless)

$\beta \quad=$ sound dispersion coefficient, $\left(c_{0} / c^{2}\right)-1$

$\gamma=$ mixture isentropic exponent (ratio of specific heats), $c_{p 0} / c_{v 0}$

$\mu \quad=$ mixture dynamic viscosity

$\rho=$ mixture density (mass per unit volume of gaseous mixture)

$\rho_{m} \quad=$ droplet mass per unit volume of mixture-droplet system

$\tau_{c} \quad=$ particle mass transfer relaxation time, $d^{2} \rho_{p} / 12 D_{0} \rho_{0}=(3 / 2) S c \tau_{d}$

$\tau_{d} \quad=$ particle dynamic (momentum) relaxation time, $d_{p}^{2} \rho_{p} / 18 \mu$

$\tau_{t} \quad=$ particle thermal (heat transfer) relaxation time, $\operatorname{Pr} c_{p p} d^{2} \rho_{p} /\left(12 \mu_{0} c_{p 0}\right)=(3 / 2) \operatorname{Pr} \tau_{d}$

$\omega \quad=$ circular frequency of sound

\section{Subscripts}

$0 \quad=$ property in undisturbed medium

$1=$ transpiration

$g \quad=$ gas (nonvolatile component, air)

$p \quad=$ droplet, particle

$s \quad=$ saturated vapor

$v \quad=$ vapor

1. Introduction

The propagation of sound (attenuation and dispersion) in a gas containing suspended droplets is of considerable interest in many important technical applications such as liquid propellant combustion chamber instability [1,2], and noise mitigation by water injection [3-7]. When a system comprised of a suspension of small spherical liquid droplets in equilibrium with a gaseous mixture is perturbed by a plane acoustic wave, fluctuations arise in the velocity, pressure, temperature and density of the gaseous mixture. Oswatitsch [8] treated sound propagation in a gas containing volatile particles exchanging heat (thus allowing evaporation/condensation), but excluded the particle viscous drag. In their classical work, Marble [9] and Marble and Wooten [10] treated sound attenuation in a saturated vapor-gas-droplet mixture (small spherical droplets initially in thermal equilibrium with the gaseous mixture) 
considering mass diffusion, momentum and heat transfer. They neglected differences between the gas and vapor properties, and assumed that the gaseous phase is both thermally and calorically perfect when mass transfer is present $[11,12]$. They also incorrectly dropped a term in the energy equation (for the mixture enthalpy) during linearization [11].

Cole and Dobbins [13] theoretically studied the attenuation of a plane acoustic wave with specific reference to air-water fogs (low droplet mass fraction), accounting for the difference between the gas constants for the vapor and the noncondensible gas, and incorporating the correct form of the energy equation for the mixture enthalpy. But they incorrectly defined the mixture enthalpy, and neglected the fluctuations of the gas constant of the inert gas-vapor mixture (retained the assumption of calorically perfect gas mixture). Their theoretical predictions deviate (overpredict) by about 35 percent from their measurements [14] for sound attenuation in atmospheric fogs (Fig. 1), largely due to the failure to account for the fluctuation in the mixture gas constant in the presence of mass transport.

Davidson[11] accounted for the individual gas constants for the vapor and the noncondensible gas (air), dispensed with the assumption that the gaseous mixture is calorically perfect, and incorporated the correct form of the linearized energy equation. With these improvements, better agreement between the theory and the experimental data of Cole and Dobbins [14] is achieved. However the agreement still fell short as evident from Fig. 1.

Gubaidullin and Ivandaev [12] took into account the transient and nonequilibrium effects of interphase mass, momentum, and energy (heat) transfer on the propagation of acoustic waves in gas-droplet mixtures. They concluded that the nonequilibrium phase transition theory has only a slight (not appreciable) improvement as the nonequilibrium curves shift the attenuation curves to the left without changing the maximum absorption coefficient appreciably. Nonequilibrium considerations with regard to the absorption and dispersion of sound were also considered by Gumerov et al. [15]. 
From the theory of convective heat and mass transfer, it is well known that transpiration tends to reduce droplet drag, and heat and mass transfer (Bird et al. [16]). Dupays [17] considered the transpiration effect on sound propagation in a droplet-gas mixture considering the general case that the droplet is in the thermal nonequilibrium with the gaseous mixture. The conservation equations of the gas-droplet mixture are essentially those of Davidson [11] except for a minor difference in the energy equation. However, their theory (either equilibrium or nonequilibrium) was not validated with any experimental data concerning the role of transpiration on sound attenuation.

The present work endeavours to investigate the role of transpiration on the attenuation and dispersion of sound in evaporating gas-droplet mixtures initially under thermodynamic equilibrium. The approach is based on an extension of the theory of Davidson [11] to accommodate transpiration on the linear relaxation processes. The results of the transpiration theory will be validated by a comparison of the predictions with the only available experimental data of Cole and Dobbins [14] for sound attenuation in atmospheric fogs. The effect of transpiration in mixtures characterized by relatively high droplet mass concentration (neglecting particle-particle interaction effects) will then be investigated with regard to sound attenuation and dispersion.

\section{Extension of Davidson's Theory for transpiration}

Considering plane wave solutions of the form

$$
\phi / \phi_{0}=\exp \left[i\left(K \omega x / c_{0}-\omega t\right)\right]
$$

Davidson [11] arrived at an expression for the dimensionless complex wave number for plane wave propagation as follows:

$$
K=k_{1}+i k_{2}=f\left(\tau_{d}, \tau_{t}, \tau_{c}, C_{m}, C_{v}, H_{p}, H_{v}, H_{g}, \operatorname{Pr}, L e, \gamma, \gamma_{v}, L, R_{v} / R_{g}\right)
$$


where

$$
\begin{gathered}
K=\left[a_{4} D_{23} /\left(a_{1} D_{23}-a_{2} D_{13}+a_{3} D_{12}\right)\right]^{1 / 2} \\
D_{i j}=b_{i} c_{j}-b_{j} c_{i} \\
a_{1}=i B /(Z-i)_{\alpha}+(1+B)\left(1-C_{v} \Omega\right) \\
a_{2}=1 \\
a_{3}=-(L-1)\left[B \Omega-i Z C_{v}(1+B)(1-\Omega)\right] \\
a_{4}=-i \gamma\left[C_{m} / \omega \tau_{d}-i-C_{m} / \omega \tau_{d}\left(1-i \omega \tau_{d}\right)\right] \\
b_{1}=G\left[i(1+\mathrm{B})\left(\left(1-C_{v} \Omega\right)\right)-B /(Z-i)\right] \\
b_{2}=C_{m} / \omega \tau_{t}+i G-i\left[H_{g}+C_{v}\left(H_{v}-H_{g}\right)\right] \\
b_{3}=-G(L-1)\left[Z C_{v}(1+B)(1-\Omega)+\Omega\right]-C_{m} /\left(\omega \tau_{1}\right) \\
c_{1}=-i W /(Z-i) \\
c_{2}=1 /\left(\omega \tau_{t} H_{p}\right) \\
W(L-1)(\Omega-1)+i-1 /\left(\omega \tau_{t} H_{p}\right) \\
B=C_{v}\left(1-R_{v} / R_{g}\right) \\
G=(\gamma-1) / \gamma, \\
Z=C_{m} / \omega \tau_{c} \\
\Omega=Z /(Z-i) \\
W=C_{v} L\left(\gamma_{v}-1\right) H_{v} /\left(\omega \tau_{c} \gamma_{v} H_{p}\right)
\end{gathered}
$$

In the preceding equation, the quantities $\tau_{d}, \tau_{t}, \tau_{c}$ denote respectively the particle relaxation times for momentum, heat and mass transfer:

$$
\tau_{d}=\frac{d_{p}^{2} \rho_{p}}{18 \mu_{0}}, \tau_{t}=\frac{d_{p}^{2} \rho_{p} c_{p 0}}{12 k_{0}}=\left(\frac{3}{2}\right) \operatorname{Pr} \tau_{d}=\frac{\tau_{c}}{L e}, \tau_{c}=\frac{\rho_{p} d_{p}^{2}}{12 D_{0} \rho_{0}}=\frac{3}{2} S c \tau_{d}=L e \tau_{t}
$$

Physically the quantities $\tau_{d}, \tau_{t}, \tau_{c}$ represent a measure of the inability of the particles to follow (respond to) the fluctuations in the fluid motion, heat and mass diffusion. The quantity $c_{p}$ denotes the mixture specific heat at constant pressure, $\rho$ the mixture density, $\mu$ the mixture viscosity, $k$ the mixture thermal conductivity, $D$ the mass diffusivity, and $d$ the diameter. The subscript 0 denotes the undisturbed state, and $p$ the particle. 
The Prandtl number Pr, Lewis number Le and the Schmidt number $S c$ of gas are defined by

$$
\operatorname{Pr}=c_{p 0} \mu_{0} / k_{0}, \quad L e=k / \rho_{0} c_{p 0} D_{0}, \quad S c=v_{0} / D_{0}=L e \operatorname{Pr}
$$

where $v=\mu / \rho$ designates the mixture kinematic viscosity. The quantities $C_{m}, C_{v}$ denote respectively the droplet loading and vapor mass loading (saturation condition) defined by

$$
C_{m}=\rho_{m 0} / \rho_{0}=n_{0} m_{p} / \rho_{0}, C_{v}=\rho_{v 0} / \rho_{0}=C_{v s}\left(T_{0}\right)
$$

where $T$ denotes the temperature, and the subscript $s$ denotes the saturation state, and $v$ for vapor. The remaining parameters are defined by

$$
H_{p}=c_{p p} / c_{p 0}, H_{v}=c_{p v} / c_{p 0}, L=h_{L 0} / R_{v} T_{0}
$$

where $c_{p p}$ is the specific heat of droplet at constant pressure, $h_{L}$ the latent heat of vaporization, and $R$ the gas constant. The energy attenuation coefficient $\alpha$ and the propagation speed $c$ are related to the complex wave number $K$ by the relation

$$
\alpha=2 k_{2}, \quad c / c_{0}=1 / k_{1}
$$

The present analysis extends the work of Davidson [11] to accommodate the effect of transpiration (so-called blowing or mass injection into the boundary layer surrounding the droplet) on the mass, momentum and heat transfer between the droplet and the surrounding gas. Without any loss of generality the propagation of sound in the presence of transpiration may be expressed by a relation similar to Eq. (2) as follows:

$$
K=K\left(\tau_{d 1 .} \tau_{11}, \tau_{c 1}\right)
$$

where the quantities $\tau_{d 1}, \tau_{t 1}, \tau_{c 1}$ correspond to the relaxation times in the presence of transpiration. They are related to the relaxation times $\tau_{d}, \tau_{t}, \tau_{c}$ in the absence of transpiration by the relations 


$$
\tau_{d 1}=\psi_{d} \tau_{d}, \quad \tau_{t 1}=\psi_{t} \tau_{t}, \quad \tau_{c 1}=\psi_{c} \tau_{c}
$$

where

$$
\psi_{d}=C_{D 1} / C_{D}, \quad \psi_{t}=N u_{1} / N u, \quad \psi_{c}=S h_{1} / S h
$$

with $C_{D 1}, N u_{1}, S h_{1}$ stand respectively for the drag coefficient, Nusselt number and Sherwood number in the presence of transpiration. In the absence of transpiration, we have

$$
C_{D}=24 / \operatorname{Re}_{p}, \quad N u=2, \quad S h=2
$$

corresponding to the Stokesian drag law, pure heat conduction limit, and pure mass diffusion limit respectively. Note that Eq. (8) is valid for low droplet Reynolds number $\left(\operatorname{Re}_{p}<<1\right.$; practically for $\mathrm{Re}_{p}$ less than about 0.1$)$. Similar modification to the particle relaxation time has been incorporated by the author $[18,19]$ to accommodate the nonlinear drag law and heat transfer.

From the theory of convective heat, mass and momentum heat transfer (Kays and Crawford [20]), the net effect of blowing (mass injection) into the boundary layer is to reduce droplet drag, heat and mass transfer. The factor $\psi_{d}$ in the presence of blowing (transpiration) is expressed by (Eisenkalm et al. [21])

$$
\psi_{d}(S)=C_{D 1} / C_{D}=1 /(1+S)
$$

where the quantity $S$ is defined by (Kays and Crawford [20])

$$
S=\left(\rho_{v s}^{\prime}-\rho_{v}\right) /\left(\rho-\rho_{v s}\right)
$$

and is usually referred to as Spalding's transport number. The following relations for $\psi_{t}, \psi_{c}$ hold in the presence of blowing based on a Couette flow approximation (Kays and Crawford [20])

$$
\psi_{i}(S)=N u_{1} / N u=\ln (1+S) / S, \quad \psi_{c}(S)=S h_{1} / S h=\ln (1+S) / S
$$

The appropriate value of $S$ typical for gas-vapor-droplet mixtures will be determined by a comparison of the theory with the experimental data of Cole and Dobbins [14]. 
From physical considerations, transpiration is expected to alter the drag, heat and mass transfer which enter into the droplet relaxation times. Its effect on other parameters such as mixture composition (less evaporation rates due to transpiration) is believed to be of secondary importance to the present problem.

\section{Results and comparison}

3.1 Comparisons with experimental data of Cole and Dobbins [14]

Figure 2a. presents a comparison of the theory with the experimental data of Cole and Dobbins [14] showing the effect of transpiration parameter $S$ on the absorption coefficient. The data are obtained in a Wilson cloud chamber in which a cloud of nearly uniform size droplets is generated. Droplet size and concentration are determined optically with the aid of Mie scattering theory for single-size droplets. The size and concentration of droplets are varied over a wide range of controlling the addition of condensation nuclei [14]. The average drop diameter ranged from $1.8 \mu m$ to $10 \mu m$ (which is about ten times larger than that in atmospheric clouds), the drop mass fraction is of the order of 0.01 , and the mixture temperature ranged from $271 \mathrm{~K}$ to $281 \mathrm{~K}$. The frequency of the disturbance is $80 \mathrm{~Hz}$. The range of average drop diameter and droplet concentration result in the value of $\omega \tau_{t} / C_{m}$ ranging from 0.4 to 16 (see Fig. 1).

The scatter (uncertainty or noise) in the attenuation data is about 15 percent in view of the difficulties associated with the optical measurement system. To the author's knowledge, these are the only quantitative measurements concerning the influence of phase transformation on sound absorption in air-water droplet system (see also Baudoin et al. [22]). This error margin is smaller than the 35 percent mismatch observed between the theory and the data of Cole and Dobbins [14].

For the present comparison, the test conditions considered correspond to those indicated by Davidson [11] in their comparisons corresponding to an average gas temperature of $T_{0}=276 \mathrm{~K}$. 


$$
\begin{aligned}
& C_{m}=0.00763, C_{v}=0.00807, \operatorname{Pr}=0.715, L e=0.839 \\
& \gamma=1.4, \gamma_{v}=1.32, R_{v} / R_{g}=1.61, H_{g}=1, H_{v}=1.9, H_{p}=4.2, L=19.7
\end{aligned}
$$

These parameters describe mean fog conditions in the experiment of Cole and Dobbins [14], see Davidson [11]. Data points are actually measured over fogs with varying values of ${ }^{\prime} C_{m}, C_{v}$ and $L$. Only data on attenuation were measured, and no data for sound dispersion were obtained.

Referring to Fig. 2a, the data shown cover a range of $\omega \tau_{t} / C_{m}$ from 0.4 to 16 . The curves for $S=0$ correspond to the case of no transpiration. It is seen that with an increase in the value of $S$ the peak attenuation (and attenuation in general) decreases, and the peak frequency increases. The maximum change in the attenuation is noticed near low frequencies characterized by $\omega \tau_{t} / C_{m} \approx 1$. The attenuation at this frequency is primarily due to phase transition (involving coupled mass and energy transfer), with the viscous and heat conduction effects being unimportant [9]. The effect of $S$ on attenuation becomes relatively small at high frequencies, characterized by $\omega \tau_{t} / C_{m}$ in excess of about 4 . At $S=0.2$, the agreement between the theory and the data appears to be most satisfactory relative to the theory of Davidson [11] in the absence of transpiration $(\mathrm{S}=0)$. Thus a value of $S=0.2$ may be regarded as representative of saturated gas-droplet-vapor system.

Figure $2 \mathrm{~b}$. shows a plot of the spectral attenuation as a function of $\omega \tau_{t}$ to reflect the effect of transpiration on attenuation due to viscous drag and heat conduction. It is seen that for the particular values of $C_{m}$ and $C_{v}$ considered, the peak attenuation due to phase transition (coupled mass and heat exchange) is considerably high (an order of magnitude larger) relative to that arising from viscous and heat conduction effects. On account of the low values of $C_{m}$, the two peaks are well separated. The values of $\omega \tau_{t}$ at which these two distinct peaks prevail are clearly indicated: the peak due to phase change is seen at $\omega \tau_{t}=C_{m}$, and that due to viscous and heat 
conduction effects at $\omega \tau_{t} \approx 1$ (Marble and Wooten [10]). For $\tau_{d} \approx \tau_{t}$, which is usually the case, the two peaks due to momentum and heat transfer nearly coincide.

Figure 3. illustrates the dependence of the dispersion coefficient $\beta=\left[\left(c_{0} / c\right)^{2}-1\right]$ on the transport parameter $S$. The predictions suggest that the effect of $S$ on the dispersion coefficient at low frequencies is appreciable. For $\omega \tau_{t} / C_{m}$ exceeding about unity, the effect of transpiration is not appreciable. It is remarkable that the strongest effect of $S$ on the dispersion is evident at the low frequency limit $\left(\omega \tau_{t} \rightarrow 0\right)$, whereas the strongest effect of $S$ on attenuation is manifested at $\omega \tau_{t} / C_{m}=1$. Unfortunately no experimental data for dispersion are available to validate the present theory.

\subsection{Predictions for high droplet concentration}

Calculations are performed for sound propagation in air-water droplet mixtures at relatively high droplet mass concentration ( $C_{m}$ ranging from 0.1 to 3 ) to reflect a range of circumstances in typical practical applications involving air-water system. Since the density of the droplet (water) is much greater than the surrounding gaseous mixture, the volume fraction of the liquid may be neglected compared with that of the gaseous species (Cole and Dobbins [14]; Temkin and Dobbins [23]). Thus interaction between neighbouring particles may be neglected even for relatively large values of the droplet mass fraction. A vapor mass loading of $C_{v}=0.1$ corresponding to $T_{0}=327 \mathrm{~K}$ (at a pressure of $0.1 \mathrm{MPa}$ ) is considered here. The relevant parameters are as follows:

$$
\operatorname{Pr}=0.7, L e=0.85, \gamma=1.4, \gamma_{v}=1.32, R_{v} / R_{g}-1.61, H_{g}=1, H_{v}=2, H_{p}=4.15, L=15.7
$$

Figure. 4 a shows the predicted attenuation with and without transpiration for $C_{m}=0.1,0.3$, 1.0 and 3.0. Two distinct peaks in attenuation are noted in the absorption spectrum for $C_{m}=0.1$ : one corresponds to phase transition, and the other due to viscous drag and heat conduction 
effects. For $C_{m}=0.1$, the attenuation peak due to phase transition exceeds that due to viscous drag and heat conduction effects; but the deviation between the two peaks is smaller than that corresponding to $C_{m}=0.01$ (Fig. 2b). For values of $C_{m}=0.3$ and higher, only one distinct peak is noticed, suggesting the existence of interaction between mass, momentum and heat transfer. The attenuation peaks increase with an increase in $C_{m}$, as is to be expected. The peak frequencies associated with the viscous drag for both $C_{m}=0.3,1.0$ and 3.0 are stationed in the neighborhood of $\omega \tau_{t} \approx 1$, but seem to increase with an increase in $C_{m}$.

With regard to the role of transpiration, the results indicate that transpiration reduces the attenuation peaks due to phase exchange and due to momentum and heat conduction. With increasing $C_{m}$, the reduction of peak attenuation in the presence of transpiration diminishes. At $C_{m}=3$, the peak absorption in the presence of transpiration is slightly enhanced. It is observed that the peak frequency increases in the presence of transpiration for all values of droplet concentrations considered. Thus the attenuation spectrum is shifted to the right as a consequence of transpiration.

Figure $4 \mathrm{~b}$. demonstrates the role of transpiration on the corresponding dispersion coefficient for various values of $C_{m}$. The results suggest that the dispersion coefficient increases with an increase in the droplet concentration $C_{m}$, as is to be expected. For frequencies characterized by $\omega \tau_{t}$ less than about 0.3 , the dispersion is relatively insensitive to transpiration. Transpiration is seen to have an appreciable effect on the dispersion of sound for $\omega \tau_{t} \approx O(1)$. Transpiration effects are seen to be very small at high frequencies $\left(\omega \tau_{t}>10\right)$. The stepwise variation in $\beta$ for the case of $C_{m}=0.1$ corresponds to the separate effects of mass transfer and viscous drag effects on the dispersion. 


\section{Discussion}

The present theory accounts for transpiration considering only monodispersion of the droplets (uniform droplet size). Another possible important mechanism affecting sound attenuation is the polydispersion of the droplets (droplet size distribution). In recent times Gubaidullin and Nigmatulin [24] and Bạudoin et al. [22] investigated polydispersion effects on sound propagation, and show that it is important to take into account polydispersion. The results of Baudoin et al. [22] suggest that the assumption of monodispersion tends to overestimate the absorption coefficient above the peak value (as observed in Fig. 2). The assumption of a mean radius tends to overestimate the attenuation, because the influence of large diameters is underestimated and the total surface area of droplets is overestimated. Both of the preceding articles recommend an average diameter $d_{31}$, based on a mean surface defined as the mean volume divided by the mean radius, is satisfactory for both attenuation and dispersion.

Thus strictly speaking, in polydispersed flow both transpiration and polydispersion effects need to be considered. In the present comparisons, the data pertain to uniform droplet size, and thus polydispersion effects are absent. Thus the exclusion of polydispersion mechanism in the present development does not invalidate the theory proposed. It is relatively simple to extend the present theory for circumstances of polydispersion by considering the recommended average droplet diameter. A detailed discussion of polydispersion effects is beyond the scope of the present investigation. Further measurements on both sound absorption and dispersion will be valuable in our understanding of the mechanisms and further development of the theoretical models for sound propagation in gas-vapor-droplet systems.

\section{Conclusion}

The effect of transpiration on sound propagation (attenuation and dispersion) in evaporating gas-vapor-droplet mixtures initially in equilibrium has been theoretically investigated. It is noted 
that at low droplet concentrations, two distinct absorption peaks due to mass transfer and viscosity-heat conduction effects are manifested, but at sufficiently high droplet concentration, significant interaction between the transport processes result in only one distinct attenuation peak. The results show that transpiration has an appreciable effect on sound attenuation and dispersion at low and high droplet concentrations. For low droplet mass fractions $\left(C_{m} \approx 0.01\right)$ when mass transfer effects predominate over viscous and heat conduction effects, the strongest effect of transpiration on sound absorption is manifested at $\omega \tau_{t} / C_{m}=1$, while the strongest effect of transpiration on sound dispersion is evident in the low frequency limit. At high drop concentrations, increases in the peak attenuation frequency and the dispersion coefficient are occasioned near $\omega \tau_{t} \approx 1$.

\section{Acknowledgment}

The author would like to thank Stanley Starr (Chief, Applied Physics Branch) of NASA Kennedy Space Center for review and helpful suggestions. 


\section{References}

[1] Yang, V., Anderson, W. (eds.), Liquid Rocket Engine Combustion Chamber Instability, Vol. 169, Progress in Astronautics and Aeronautics, AIAA, Washington, D.C., 1995.

[2] Summerfield, M., and Krier, H., Role of aluminium in suppressing instability in solid propellant rocket motors, Problems of Hydrodynamics and Continuum Mechanics, Sixtieth Anniversary Volume, Society for Independent and Applied Mathematics, Philadelphia, PA, 1969, pp. 703-717.

[3] Kandula, M., Broadband shock noise reduction in turbulent jets by water injection, Applied Acoustics 2009;70:1009-1014.

[4] Kandula, M., Prediction of turbulent jet mixing noise reduction by water injection, AIAA Journal 2008; 46:2714-2722.

[5] Krothapalli, A., Venkatakrishnan, L., Lourenco, L., Greska, B., Elavarasan, R., Turbulence and noise suppression of a high-speed jet by water injection, J. Fluid Mechanics 2003;491:131-59.

[6] Mahanta, P.K., Vajpayee, S., Sadek, M.M., Suspended droplets as a means of noise reduction, Applied Acoustics 1986;19(2)77-90.

[7] Leighton, T.G., Jiang, J., Baik, K., A TV demonstration of sound absorption connecting the space shuttle to submarines, Acoustics Bulletin 2011;36(4)35-40.

[8] von Oswatitsch, K., Die Dispersion und Absorption des Schlles in Wolken (Sound dispersion and absorption in clouds), Physik. Zeits. 1941; 42: 365-378 (translated as NSAS-TT-F-10939).

[9] Marble, F.E, Some gas dynamic problems in the flow of condensing vapors, Astronautica Acta $1969 ; 14: 585-614$.

[10] Marble, F.E., Wooten, D.C., Sound attenuation in a condensing vapor, Physics of Fluids $1970 ; 13: 2657-2664$. 
[11] Davidson, A., Sound propagation in fogs, Journal of the Atmospheric Sciences 1975; 32 (11):2201-2205.

[12] Gubaidullin, A., Ivandaev, A.I., Influence of phase transitions on sound propagation in fogs: comparison of theory and experiment," Translated (by Tyumen) from Prikladnoi Mekhaiki i Tekhnicheskoi Fiziki (Journal of Applied Mechanics and Technical Physics) $1990 ; 6: 27-34$

[13] Cole, J.E., Dobbins, R.A., Propagation of sound through atmospheric fog, Journal of the Atmospheric Sciences 1970; 27: 426-434.

[14] Cole, J.E., Dobbins, R.A., Measurements of attenuation of sound by a warm air fog, Journal of the Atmospheric Sciences 1971; 28:202-209.

[15] Gumerov, N.A., Ivandaev, A.I., Nigmatulin, R.I., Sound waves in a monodisperse gasparticle or vapor-droplet mixtures, Journal of Fluid Mechanics 1988; 193:53-74.

[16] Bird, B., Stewart, W.E., Lightfoot, E.N., Transport Phenomena. New York: John Wiley and Sons; 1960.

[17] Dupays, J.; Mass transfer effects on sound propagation in droplet-gas mixture, in Combustion of Énergetic Materials (eds: Kuo and Deluca), Begell House; 2002.

[18] Kandula, M., Spectral attenuation of sound in dilute suspensions with nonlinear particle relaxation, Journal of the Acoustical Society of America 2008; 124(5):EL284-290.

[19] Kandula, M., Dispersion of sound in dilute suspensions with nonlinear particle relaxation, Journal of the Acoustical Society of America 2010;127(3):EL115-120.

[20] Kays, W.M., and Crawford, M.E., Convective Heat and Mass Transfer. $2^{\text {nd }}$ ed. New York: McGraw-Hill;1980.

[21] Eisenklam, P., Arunachalam, S.A., Weston, J.A., Evaporation rates and drag resistance of burning drops, Proceedings of the 11th Symposium (International) on Combustion, Combustion Institute, Pittsburgh, PA, 1967; pp. 715-728. 
[22] Baudoin, M., Coulouvrat, F., Thomas, J-L., Sonic boom absorption by atmospheric clouds, J. Acoustical Society of America 2011;130(3):1142-1153.

[23] Temkin, S., Dobbins, R.A., Attenuation and dispersion of sound by particulate Relaxation Processes, Journal of the Acoustical Society of America 1966; 40(2):317-324.

[24] Gubaidullin, D.A., Nigmatulin, R.I., On the theory of acoustic waves in polydispersed gasvapor-droplet suspensions, Int. J. Multiphase Flow 2000;26:207-228. 


\section{Figure Captions}

Fig. 1. Comparison of dimensionless attenuation coefficient from Davidson's theory with the experimental data of Cole and Dobbins [14].

Fig. 2. (a) Comparison of dimensionless absorption coefficient from transpiration theory with data of Cole and Dobbins [14]. (b) Comparison of the dimensionless absorption coefficient with $\omega \tau$, from transpiration theory with the data of Cole and Dobbins [14].

Fig. 3. Predicted dispersion coefficient from transpiration theory for the test conditions of Cole and Dobbins [14].

Fig. 4 (a) Effect of transpiration on dimensionless absorption coefficient predicted by the present theory for high droplet concentrations. (b) Effect of transpiration on the dispersion coefficient predicted by the present theory for high droplet concentrations. 


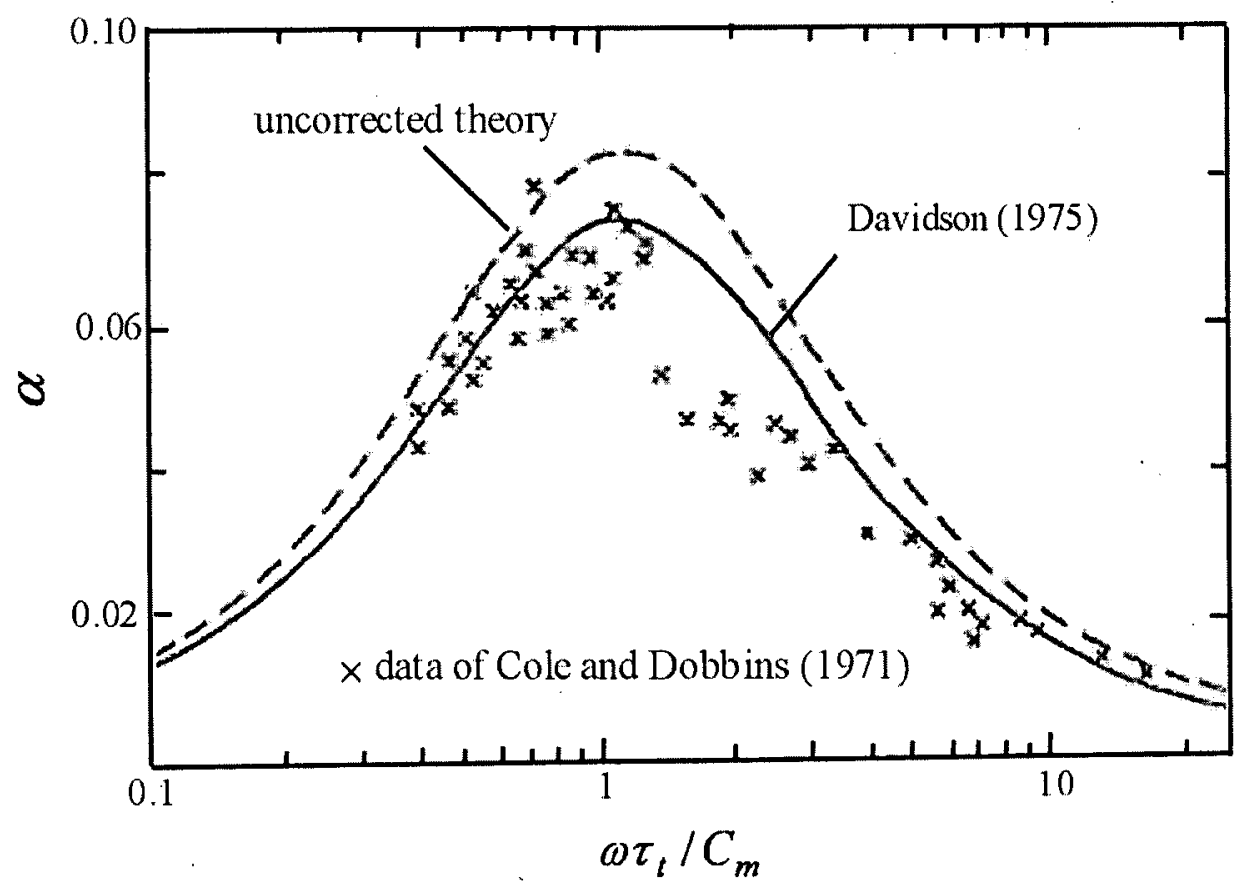

Fig. 1. Comparison of dimensionless attenuation coefficient from Davidson's theory with the experimental data of Cole and Dobbins [14]. 


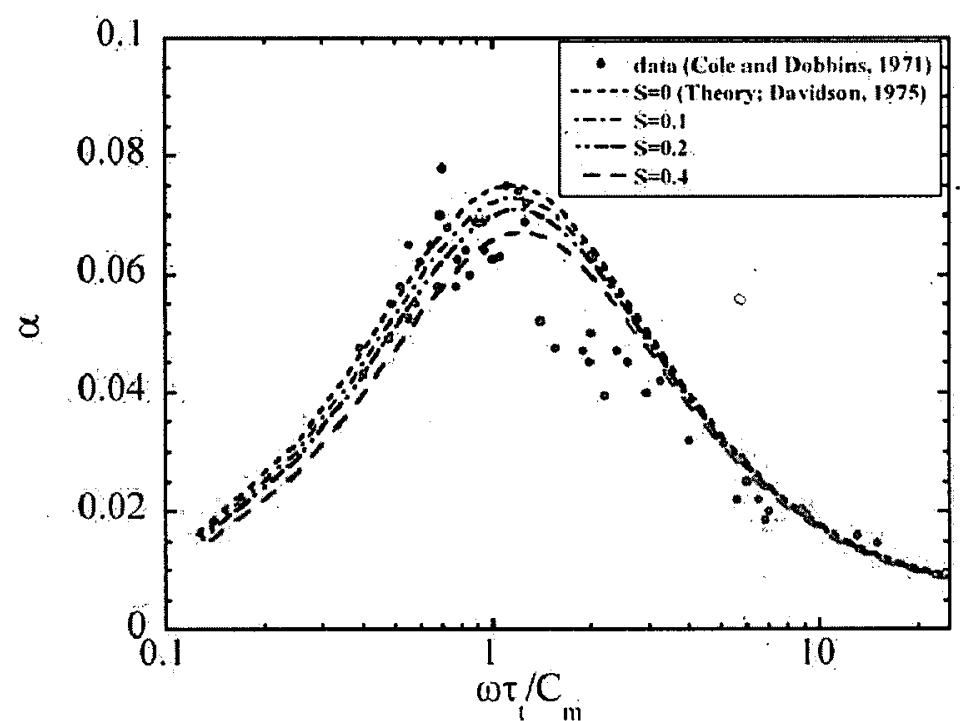

(a)

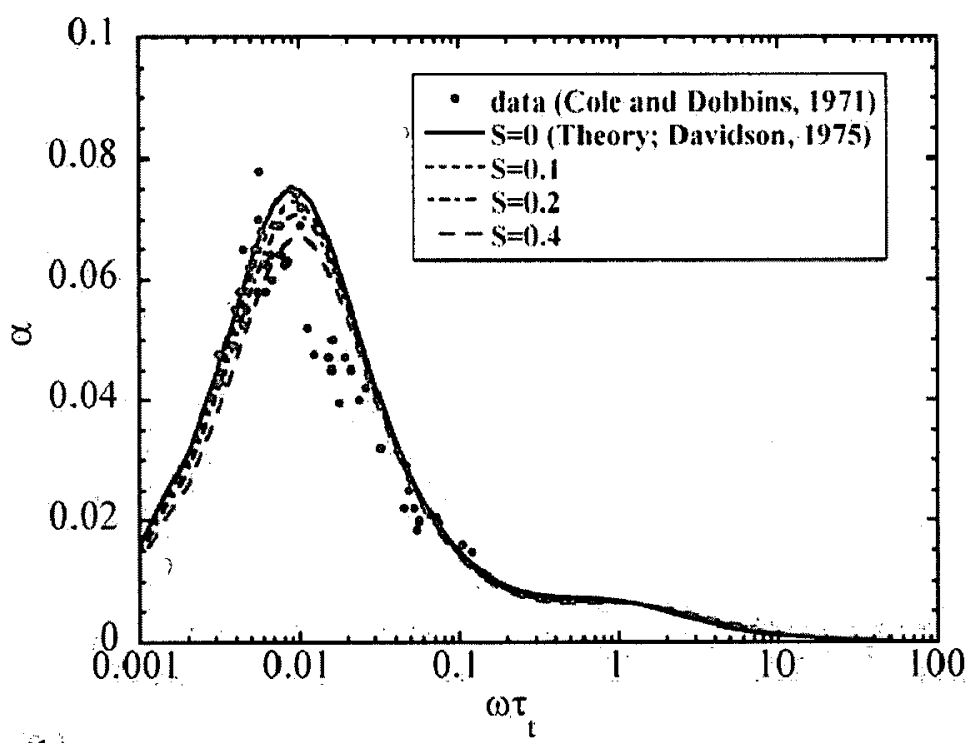

(b)

Fig. 2. (a) Comparison of dimensionless absorption coefficient from transpiration theory with data of Cole and Dobbins [14], (b) Comparison of dimensionless absorption coefficient with $\omega \tau$, from transpiration theory with the data of Cole and Dobbins [14] 


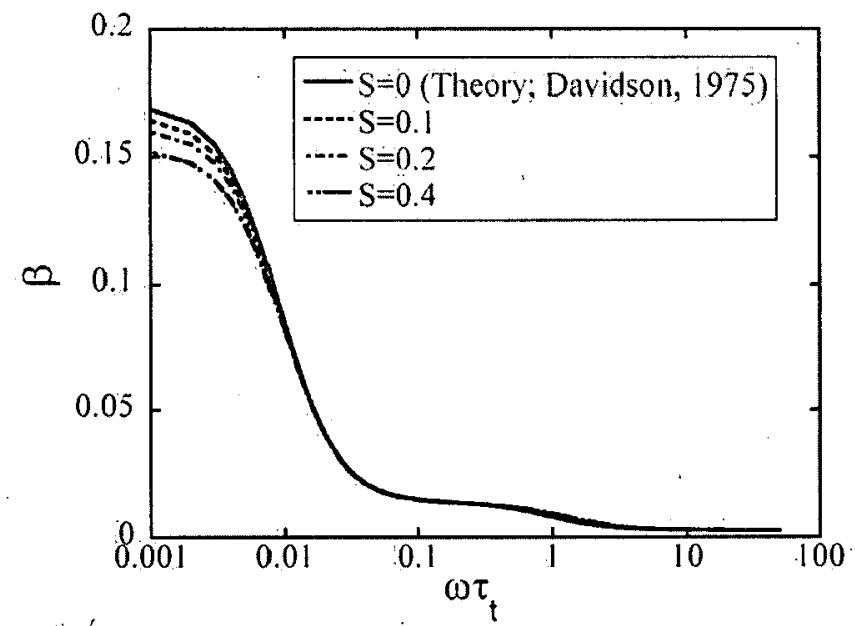

(a)

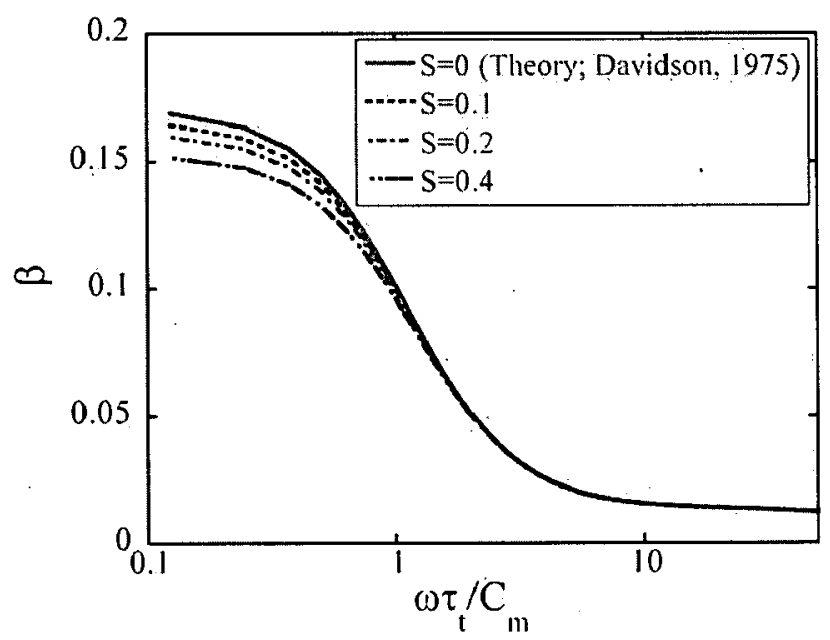

(b)

Fig. 3. Predicted dispersion coefficient from transpiration theory for the test conditions of Cole and Dobbins [14]. 


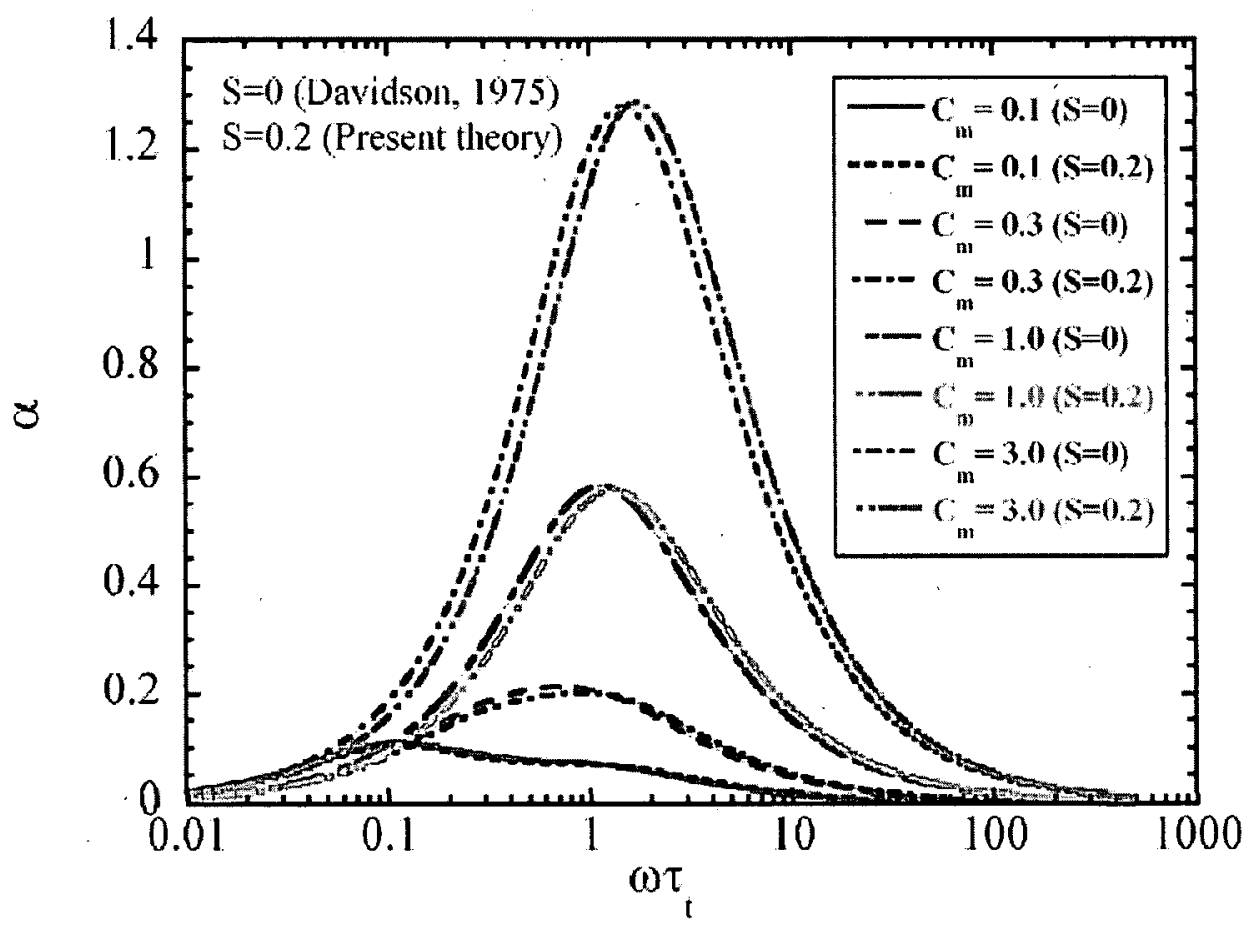

(a)

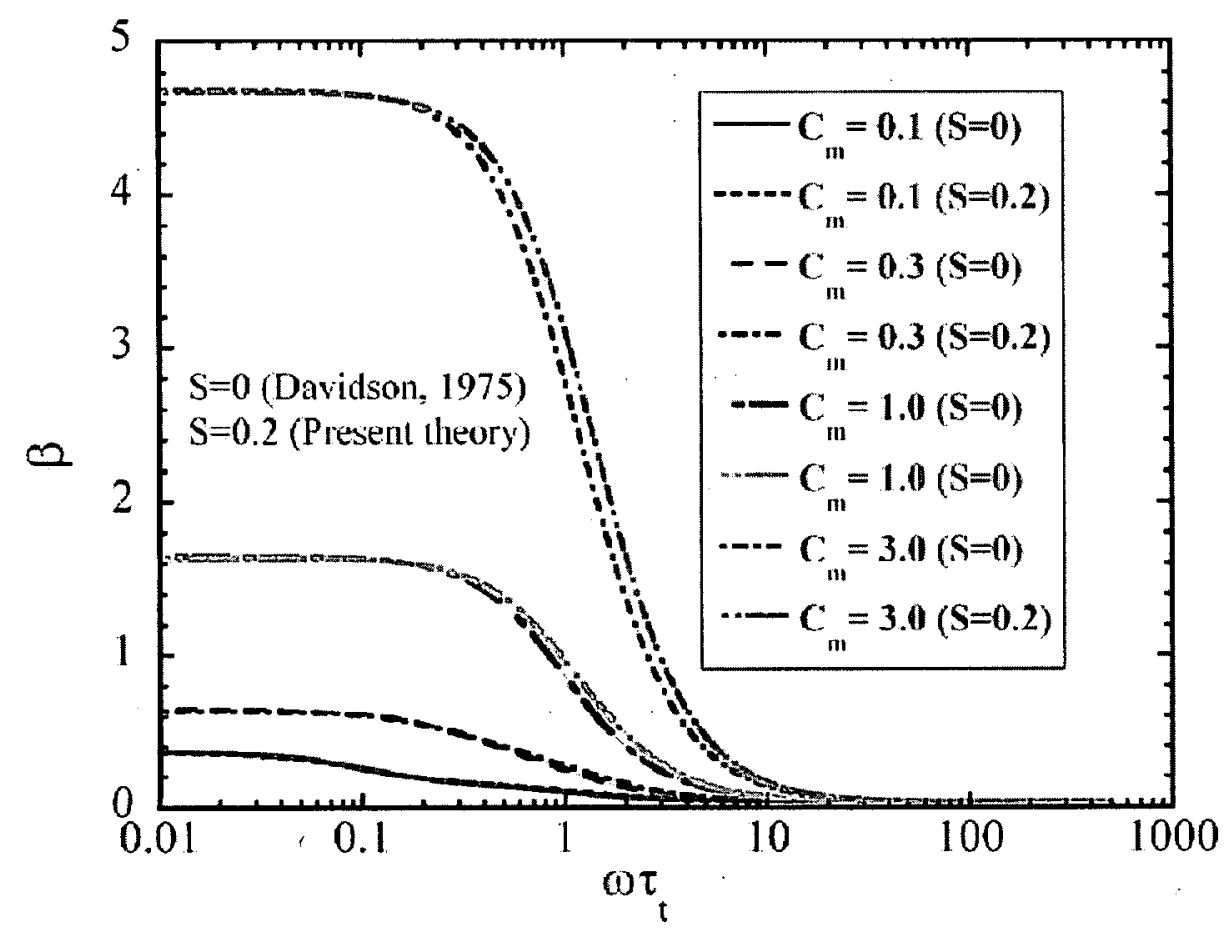

(b)

Fig. 4 (a) Effect of transpiration on dimensionless absorption coefficient predicted by the present theory for high droplet concentrations. (b) Effect of transpiration on the dispersion coefficient predicted by the present theory for high droplet concentrations. 